\title{
Dynamic range expansion by gas-phase ion fractionation and enrichment for imaging mass spectrometry
}

\author{
Boone M. Prentice*1,2, Daniel J. Ryan3, Kerri J. Grove2,3, D. Shannon Cornett4, Richard M. \\ Caprioli1,2,3,5, Jeffrey M. Spraggins $1,2,3$ \\ 1Department of Biochemistry, 2 Mass Spectrometry Research Center, and \\ 3Department of Chemistry, Vanderbilt University, Nashville, TN 37232; Bruker Daltonics, \\ Billerica, MA 01821; 5Department of Pharmacology and Medicine, Vanderbilt University \\ Medical Center, Nashville, TN 37232
}

Running title: Quadrupole-based ion enrichment for imaging mass spectrometry

*Address correspondence to:

Dr. B.M. Prentice

214 Leigh Hall, PO Box 117200

Department of Chemistry

University of Florida

Gainesville, FL 32608, USA

Phone: (352) 392-0556

Fax: $\quad$ (352) 392-4651

E-mail: booneprentice@chem.ufl.edu 


\section{ABSTRACT}

In the analysis of biological tissue by imaging mass spectrometry (IMS), the limit of detection and dynamic range are of paramount importance in obtaining experimental results that provide insight into underlying biological processes. Many important biomolecules are present in the tissue milieu in low concentrations and in complex mixtures with other compounds of widely ranging abundances, challenging the limits of analytical technologies. In many IMS experiments, the ion signal can be dominated by a few highly abundant ion species. On trap-based instrument platforms that accumulate ions prior to mass analysis, these high abundance ions can diminish the detection and dynamic range of lower abundance ions. Herein, we characterize two strategies for combating these challenges during IMS experiments on a hybrid QqFT-ICR MS. In one iteration, the mass resolving capabilities of a quadrupole mass filter are used to selectively enrich for ions of interest via a technique previously termed continuous accumulation of selected ions (CASI). Secondly, we have introduced a supplemental dipolar AC waveform to the quadrupole mass filter of a commercial QqFT-ICR mass spectrometer to perform selected ion ejection prior to the ion accumulation region. This setup allows the selective ejection of the most abundant ion species prior to ion accumulation, thereby greatly improving the molecular depth with which IMS can probe tissue samples. 


\section{INTRODUCTION}

The limit of detection and dynamic range of a mass spectrometry experiment are of critical importance when analyzing complex biological mixtures. These analytical figures of merit determine the ability of an experiment to comprehensively study complex cellular pathways. Proteins, lipids, and metabolites in biological samples can easily span over six orders of magnitude in concentration, testing the limits of analytical mass spectrometry.1 Many proteomics, lipidomics, and metabolomics studies leverage condensed phase fractionation technologies, such as liquid chromatography (LC-MS) and capillary electrophoresis (CE-MS), prior to mass spectrometric analysis to help separate and simplify these complex mixtures.2-5 Orthogonal compound separation by chromatography prior to mass spectrometry has been used for decades to improve peak capacity.6-10 Unfortunately, the longer analysis times required by these hyphenated techniques preclude their use in molecular imaging workflows.11

Matrix-assisted laser desorption/ionization (MALDI) has emerged as the most common imaging mass spectrometry (IMS) approach for the in situ molecular interrogation of tissue samples. In MALDI IMS, ions generated from the tissue surface by laser irradiation are sampled directly into the mass spectrometer.12-15 Thousands of molecular species can be detected in a single imaging mass spectrometry analysis.16 However, many compounds of interest in the tissue milieu are present in low abundance and are thus undetectable using conventional IMS methods. Advanced sample preparation protocols, including tissue washing and chemical derivatization, have been used to improve sensitivity for a desired analyte or class of analytes.17-23 However, these strategies can be time consuming and may introduce tissue deformation and/or analyte delocalization within the tissue. Instead, gas-phase separation techniques represent rapid and 
flexible means to fractionate complex mixtures of biomolecules after ion generation from the tissue surface and prior to MS detection.

Gas-phase separation and accumulation approaches have been employed in various configurations to improve analytical performance. For example, ion mobility-mass spectrometry (IM-MS) has been shown to improve sensitivity and selectivity by fractionating mixtures on the basis of ion collisional cross section (CCS).24-26 Other approaches have taken advantage of hybrid mass spectrometry instrumentation to perform ion accumulation in a separate ion trap prior to mass analysis.27 For example, ion accumulation in a linear octopole ion trap positioned external to a Fourier transform ion cyclotron resonance (FTICR) cell enabled dramatic improvements in sensitivity.28 29 These external accumulation setups have demonstrated limits of detection of 20 zmol of cytochrome $c$ and duty cycles of $100 \%$ during electrospray ionization (ESI) experiments.

Despite the power of external accumulation methods to maximize sensitivity, there is still a wide dynamic range of molecular concentrations that limit the achievable sensitivity. One or more highly abundant compounds can often preclude the detection of more lowly abundant ions in the mass spectrum. The ability to selectively enrich for only the ions of interest while discarding other ion signals would thus maximize the sensitivity for the desired compound, especially on trapbased mass spectrometry platforms. One way to perform this involves the use of a quadrupole mass filter (QMF) to restrict ion transmission to a narrow mass range prior to accumulation in an ion trap.30, 31 This mass isolation followed by accumulation can be termed 'mass-selective ion accumulation' and has been commonly employed on hybrid mass spectrometers.32, 33 Conversely, the quadrupole mass filter can also be used to perform selected ejection of one or more highly abundant ions, allowing for additional broadband accumulation of all of the more lowly abundant compounds, up to the charge capacity of the trap.34 This 'selected ion ejection' (SIE) has been 
achieved via resonant ejection using a supplemental dipolar AC excitation waveform as the ions traverse the QMF.35-37 This has been demonstrated to improve the sensitivity of LC-MS proteomics experiments performed on a hybrid QqFT-ICR MS.

Several gas phase fractionation approaches have been adapted to improve sensitivity and dynamic range in imaging mass spectrometry experiments. Ion mobility has been used following both MALDI and desorption electrospray ionization (DESI) to improve the detection of low abundance ions and to separate isobaric (i.e., same nominal mass) compounds. $38-43$ We and others have described preliminary uses of selective ion accumulation during MALDI IMS to improve limits of detection in a range of applications.44-51 This has largely been performed using a targeted quadrupole mass filter isolation window on hybrid QqFT-ICR mass spectrometers and has been termed continuous accumulation of selected ions (CASI). Herein, we seek to characterize the sensitivity and dynamic range enhancements afforded by CASI IMS. Additionally, we have implemented selected ion injection on a hybrid QqFT-ICR for IMS. The supplementary AC signal applied to the quadrupole enables a small $\mathrm{m} / \mathrm{z}$ interval to be ejected as the ions transmit through the quadrupole mass filter, allowing for the broadband enrichment of ions of interest downstream in the hexapole accumulation cell. Importantly, both the CASI and SIE ion accumulation methods allow for ions to be filtered in the quadrupole prior to their admittance into the hexapole trap and eventual transfer to the ICR cell for mass analysis, thereby improving measurement sensitivity and dynamic range for user-defined $m / z$ intervals. 


\section{EXPERIMENTAL}

Materials

2,5-dihydroxyacetphenone (DHA), 1,5-diaminonapthalene (DAN), red phosphorous, hematoxylin stain, glycerol, and aluminum potassium sulphate were purchased from SigmaAldrich Chemical Co. (St. Louis, MO, USA). Ethanol and methanol were purchased from Fisher Scientific (Pittsburgh, PA, USA).

\section{Mass Spectrometry}

CASI experiments were performed on a $15 \mathrm{~T}$ solariX FT-ICR mass spectrometer and selected ion ejection experiments were performed on a 9.4T solariX FT-ICR mass spectrometer, both equipped with an Apollo II dual MALDI/ESI source. The 15T instrument was equipped with an infinity cell and the 9.4T instrument with a dynamically harmonized ParaCell52 (Bruker Daltonics, Billerica, MA). The MALDI source utilizes a Smartbeam II Nd:Yag laser (2 kHz, 355 nm). A 2416A arbitrary waveform generator (Pragmatic Instruments, San Diego, CA) has been successfully coupled to the quadrupole mass filter using a center-tapped transformer (Supplemental Figure 1). The transformer was hand-wound using a $10 \mathrm{~mm} \mathrm{ID,} 18 \mathrm{~mm}$ OD ferrite core. The center tap of the secondary winding is attached to the existing instrument vacuum feedthrough that provides the signal from the drive RF circuit for one of the pairs of rods on the quadrupole array. The secondary winding connections are attached to opposing rods of one rod pair. The primary winding connections are attached to vacuum feedthroughs that have been installed on a flange of the quadrupole housing. These feedthroughs are connected to the arbitrary waveform generator and allow a single, user-defined AC signal to be applied to the quadrupole mass filter. The primary and secondary windings of the transformer each have 7 turns, which 
minimized the inductive load on the instrument RF circuit while still allowing for roughly $100 \%$ supplementary waveform coupling efficiency across the transformer. This setup allows for the maximum RF voltage to be applied without overdrawing the current on the power supply. Importantly, no changes in ion transmission efficiency were observed due to the new hardware modifications. Agilent tune mix was used to verify consistent ion signal across a broad mass range. This was verified in both positive ion and negative ion modes (Supplemental Figure 2). This ensures that no detrimental effects on ion transmission efficiency have been caused by the modification, allowing for normal instrument operation when selected ion ejection experiments are not being performed. For selected ion ejection experiments, the secular frequency of an ion of interest was calculated based on the dimensionless Mathieu trapping parameters and using the Dehmelt approximation. Briefly, the known operating conditions of the quadrupole (i.e., physical dimensions, operating frequency, and voltages) allow for the ion secular frequency to be calculated. This frequency was then manually programmed into the arbitrary waveform generator.

\section{Lipid Extraction}

Microscope slides with uniform lipid coatings were prepared using a TM Sprayer (HTX Technologies, LLC, Chapel Hill, NC, USA). Briefly, the bulk lipid content was extracted from rat brain tissue sections $(\sim 30 \mathrm{mg})$ using an adapted Folch method.53 A $20 \mu \mathrm{L}$ aliquot of a chloroform/methanol $(2: 1 \mathrm{v} / \mathrm{v})$ solution was used for each milligram of tissue. The tissue was pulverized and sonicated for 5 minutes. A $400 \mu \mathrm{L}$ aliquot of distilled water was then added and the mixture vortexed and centrifuged for 5 minutes at 3,000 rpm. A $200 \mu \mathrm{L}$ aliquot of the lower phase (organic) that contained the bulk lipid content was removed and added to $5 \mathrm{~mL}$ of a DAN MALDI matrix solution $(15 \mathrm{mg} / \mathrm{mL}$ in $90 \%$ acetonitrile). A uniform coating of this lipid/matrix 
mixture was applied to a glass slide using a TM Sprayer (flow rate: $0.15 \mathrm{~mL} / \mathrm{min}$, nitrogen flow: $10 \mathrm{psi}$, spray temperature: $30^{\circ} \mathrm{C}, 4$ passes with offsets and rotations, spray velocity: $1300 \mathrm{~mm} / \mathrm{min}$ ) (HTX Technologies, LLC, Chapel Hill, NC, USA).

\section{Imaging Mass Spectrometry}

Control rat brain was purchased from Pel-Freeze Biologicals (Rogers, AR, USA) and stored at $-80^{\circ} \mathrm{C}$. Transverse sections of rat brain were prepared using a Cryostartm NX70 Cryostat (Thermo Fisher Scientific, San Jose, CA, USA) and thaw mounted onto indium tin oxide (IT)coated slides. For CASI lipid analysis, a DAN matrix layer was applied using a home-built sublimation apparatus $\left(110^{\circ} \mathrm{C},<70\right.$ mtorr, 7 minutes $) .54,55$ For SIE lipid analyses, a DHA matrix layer was applied using a home-built sublimation apparatus $\left(110^{\circ} \mathrm{C},<70 \mathrm{mtorr}, 4.5\right.$ minutes $)$.

CASI images were acquired using a raster step of $100 \mu \mathrm{m}$ in both the $\mathrm{x}$ and $\mathrm{y}$ dimensions using a $40 \mu \mathrm{m}$ diameter laser beam. Data were collected from $\mathrm{m} / \mathrm{z}, 300$ to $\mathrm{m} / \mathrm{z}, 2000$ in negative ion mode using 512k free induction decay (FID) time. SIE images were acquired using a raster step of $100 \mu \mathrm{m}$ in both the $\mathrm{x}$ and $\mathrm{y}$ dimensions using a $25 \mu \mathrm{m}$ diameter laser beam. Data were collected from $\mathrm{m} / z 500$ to $\mathrm{m} / z, 2,000$ in negative ion mode using a 256k FID. The full scan and CASI/SIE image comparisons were collected in a single imaging run in order to eliminate the need for normalization between separate images (i.e., the image analysis was paused in FlexControl midway through the run and the CASI or SIE functionality was manually enabled before then restarting the image run). The resulting ion images were visualized in FlexImaging 5.0 (Bruker Daltonics, Billerica, MA). One half of the rat brain sections are imaged with either CASI or selected ion ejection enabled and one half of the rat brain sections are imaged with CASI or selected ion ejection disabled to allow for direct molecular comparisons within the same image on 
the same tissue section. Lipids were identified by searching high resolution accurate mass measurements (HRAM) against the LIPID MAPS (Lipidomics Gateway, www.lipidmaps.org) online database and are reported using total carbon : double bond (TC:DB) sum composition nomenclature. Data analysis was performed using DataAnalysis and Compass IsotopePattern (Bruker Daltonics) as well as the mMass software package.56

\section{RESULTS}

\section{Selective Ion Accumulation}

Continuous accumulation of selected ions (CASI) has been used by us and others to improve the limit of detection in a variety of IMS applications.44-51 We seek here to analytically characterize the level of sensitivity and dynamic range improvements that can be achieved by CASI IMS. CASI on the hybrid QqFT-ICR mass spectrometer leverages a pair of multipole devices prior to the ICR cell to perform selective ion accumulation (Figure 1a). Ions generated from the MALDI source pass through a series of transfer ion optics before reaching a quadrupole mass filter (QMF). Operating the QMF in RF/DC mode allows for the transmission of ions within a select $m / z$ window. Ions exiting the QMF are accumulated in a hexapole ion trap. This process continues for a user-defined number of MALDI laser shots, allowing the hexapole ion trap to be filled with the targeted analytes. The accumulated ions are then collectively transferred to the ICR cell where mass analysis is performed. 


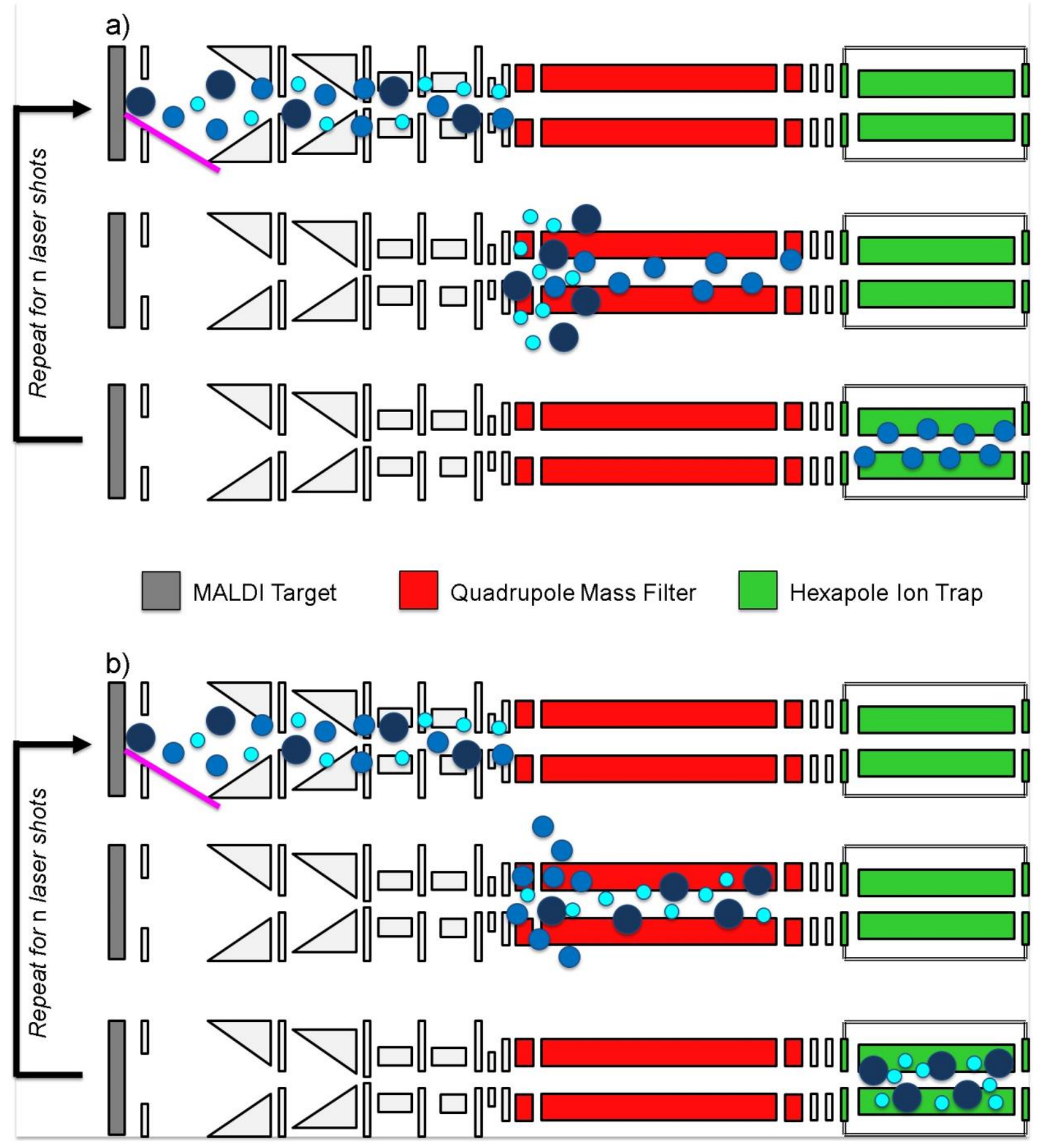

Figure 1: Scheme showing (a) the isolation of a small $\mathrm{m} / \mathrm{z}$ window by CASI and (b) the selected ion ejection of a small $\mathrm{m} / \mathrm{z}$ range. Source ion optics, including ion funnels and transfer multipoles, are shown in light grey. Instrument not drawn to scale and components have been omitted for simplicity, such as the ESI and APCI sources as well as the ICR cell and related transfer ion optics. 
The CASI process is a gas-phase enrichment method that maximizes the signal intensity for selected ions at the expense of ions with $m / z$ values outside of the QMF transmission window. Given the finite charge capacity of the hexapole ion trap, continually injecting ions across a broad mass range quickly fills the trap (it should be noted that the downsteam ICR cell also has a space charge limit that if breached will result in peak distortion, which often occurs at smaller number of ions than the storage limit of the hexapole).57-60 This improved performance using the CASI workflow demonstrated by performing MALDI analysis of a lipid extract from rat brain tissue. As shown in Figure 2, accumulation across the entire lipid mass range fills the hexapole ion trap in roughly 2,000 laser shots (orange circles). Accumulation of ions from successive laser shots quickly surpasses the space charge limit of the trap, as is embodied by the decreasing abundance of $m / z$ 734.56. Conversely, the CASI experiment restricts the QMF transmission window to $m / z$ $734.5 \pm 2.5 \mathrm{Da}$, dramatically decreasing the total ion flux transmitted into the hexapole (blue crosses in Figure 2). This targeted method now primarily fills the hexapole ion trap with the $\mathrm{m} / \mathrm{z}$ 734.56 ion of interest. The ion accumulation can now persist for additional laser shots before reaching the charge capacity of the trap. A maximum signal-to-noise ratio $(\mathrm{S} / \mathrm{N})$ of roughly 1,600 is reached after 8,000 shots, an over 5-fold improvement in $\mathrm{S} / \mathrm{N}$ compared to the full scan experiment that roughly correlates with the increased number of laser shots. Improvements in $\mathrm{S} / \mathrm{N}$ of over 40-fold can readily be achieved with the CASI method (Supplemental Figure 3). The dynamic range is also clearly improved by similar relative magnitudes. This process improves $\mathrm{S} / \mathrm{N}$ linearly by accumulating additional ion signal (i.e., "S") and is a more efficient means for improving $\mathrm{S} / \mathrm{N}$ than spectral averaging, which only improves $\mathrm{S} / \mathrm{N}$ by the square-root of the number of averaged scans (green squares in Figure 2). 


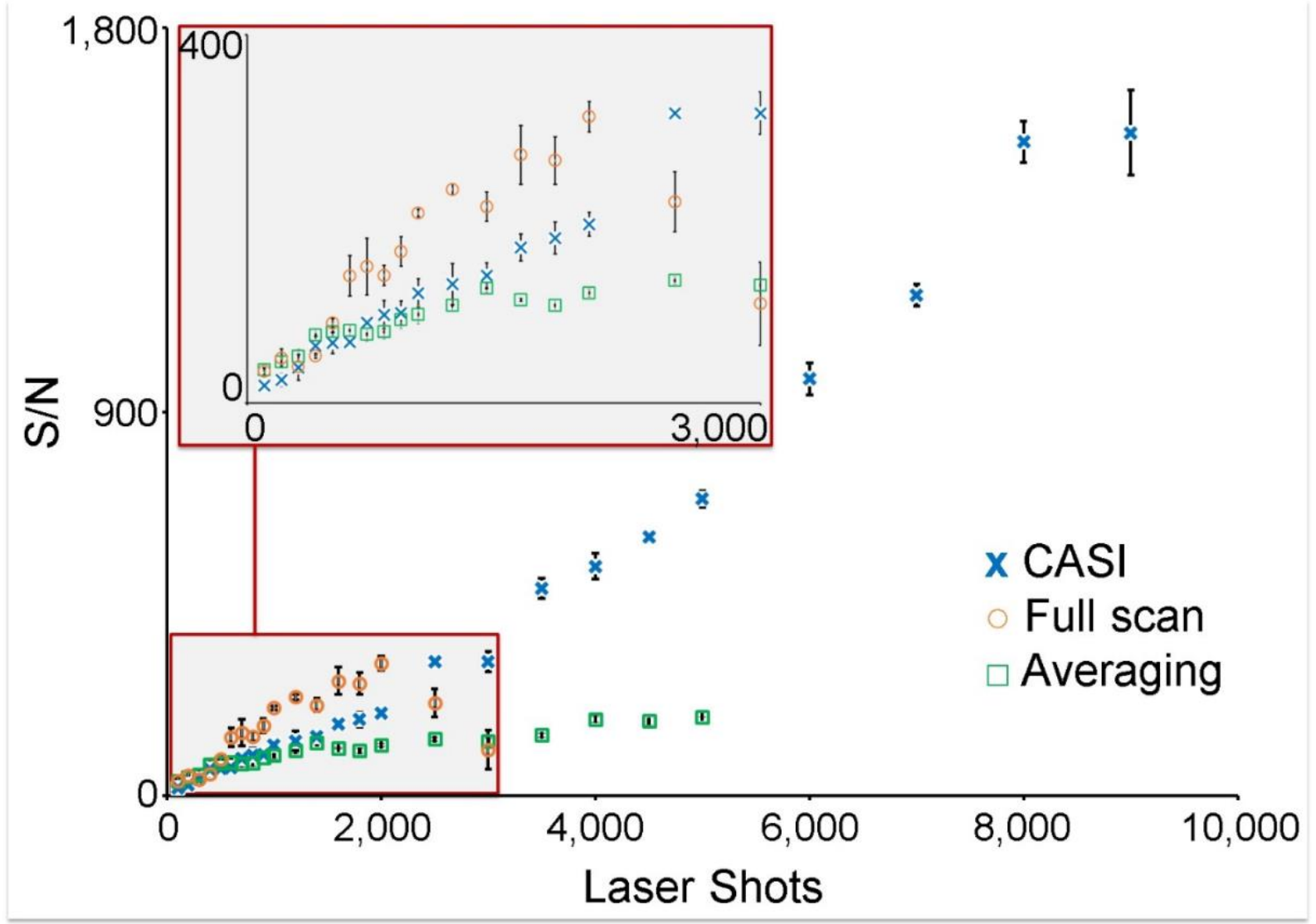

Figure 2: The $\mathrm{S} / \mathrm{N}$ of $m / z 734.56$ from a rat brain lipid extract is shown as a function of the number of MALDI laser shots for the full scan (orange circles), a $5 \mathrm{Da}$ isolation window CASI (blue crosses), and spectral averaging (green squares) acquisition modes. Each measurement represents four averages.

In an imaging mass spectrometry experiment, CASI can be used to improve the detection limit of lowly abundant endogenous and exogenous compounds in the tissue. This is exemplified using a lipid analysis of rat brain (Figure 3 and Figure 4). A full scan mass spectrum necessitated restricting the number of laser shots to 100 shots per pixel in order to stay within the charge capacity. Using a QMF isolation window of \pm 37.5 Da centered at $m / z$ 845, the CASI experiment allowed for the accumulation of 1,000 laser shots without reaching the space charge limit, showing markedly improved ion intensities within the selected mass range (Figure 3b). Several ions below the detection limit in the full scan measurement are now observed in CASI experiment (Figure 
3d). These improvements in ion intensities translate into improvements in image intensity and quality (Figure 4). Many lipids within the selected mass range were of low abundance or not detectable (left hemisphere of the rat brain ion images shown in Figure 4). However, these lipids are readily detected with strong image contrast (i.e., dynamic range) in the CASI imaging experiment (right hemisphere of the rat brain ion images shown in Figure 4).

In order to enable the accumulation of additional laser shots the "random smart walk" MALDI functionality was enabled during CASI experiments. For the experiment shown in Figure 4, the smart walk performs a random target raster for 1,000 laser shots within a $100 \mu \mathrm{m}$ by $100 \mu \mathrm{m}$ square pixel using a $40 \mu \mathrm{m}$ diameter laser beam. This more thoroughly ablates all of the material within the pixel compared to the full scan experiment that did not use the random walk raster setting; instead, all 100 shots per pixel were fired at a single location within the $100 \mu \mathrm{m}$ by 100 $\mu \mathrm{m}$ pixel using the $40 \mu \mathrm{m}$ diameter laser beam, leaving a portion of the pixel unablated. In order to accumulate sufficient ions for an increase in sensitivity during a CASI experiment, a target raster may or may not be required depending on the experimental conditions (e.g., matrix thickness, laser power, width of the CASI isolation window, intensity of ions within the CASI window, etc.). It should be noted that a target raster will sample a larger area of the tissue, which may require sacrificing spatial resolution in some instances (i.e., increasing the raster step size between pixels). 
a)

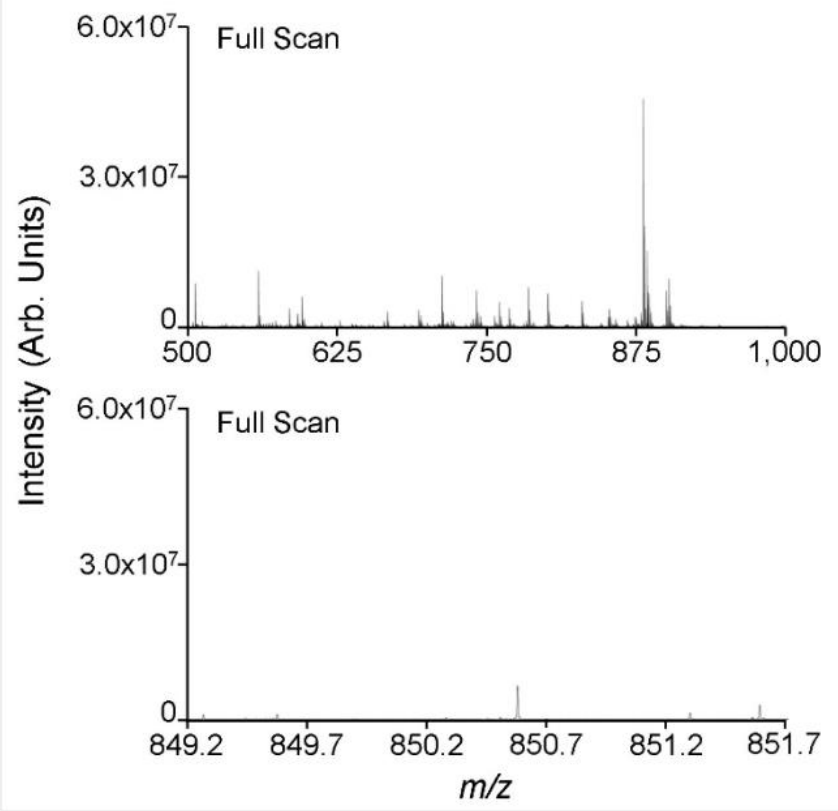

b)

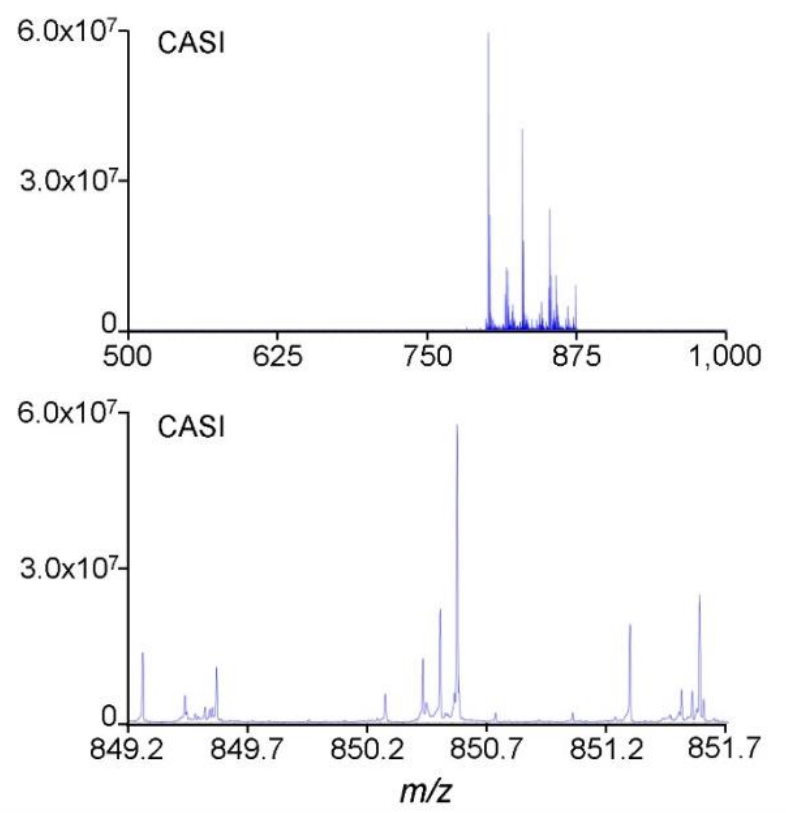

Figure 3: Lipid analysis of rat brain in (a) full scan acquisition mode and (b) using a 75 Da CASI window centered at $\mathrm{m} / \mathrm{z}, 845$. Spectral zoom-ins of a $2.5 \mathrm{Da} \mathrm{m} / \mathrm{z}$ range are included to demonstrate the improvement in ion detection using CASI. The full scan experiment employed 100 laser shots/pixel with the smart walk feature disabled and the CASI experiment employed 1,000 laser shots/pixel with the smart walk feature enabled. 


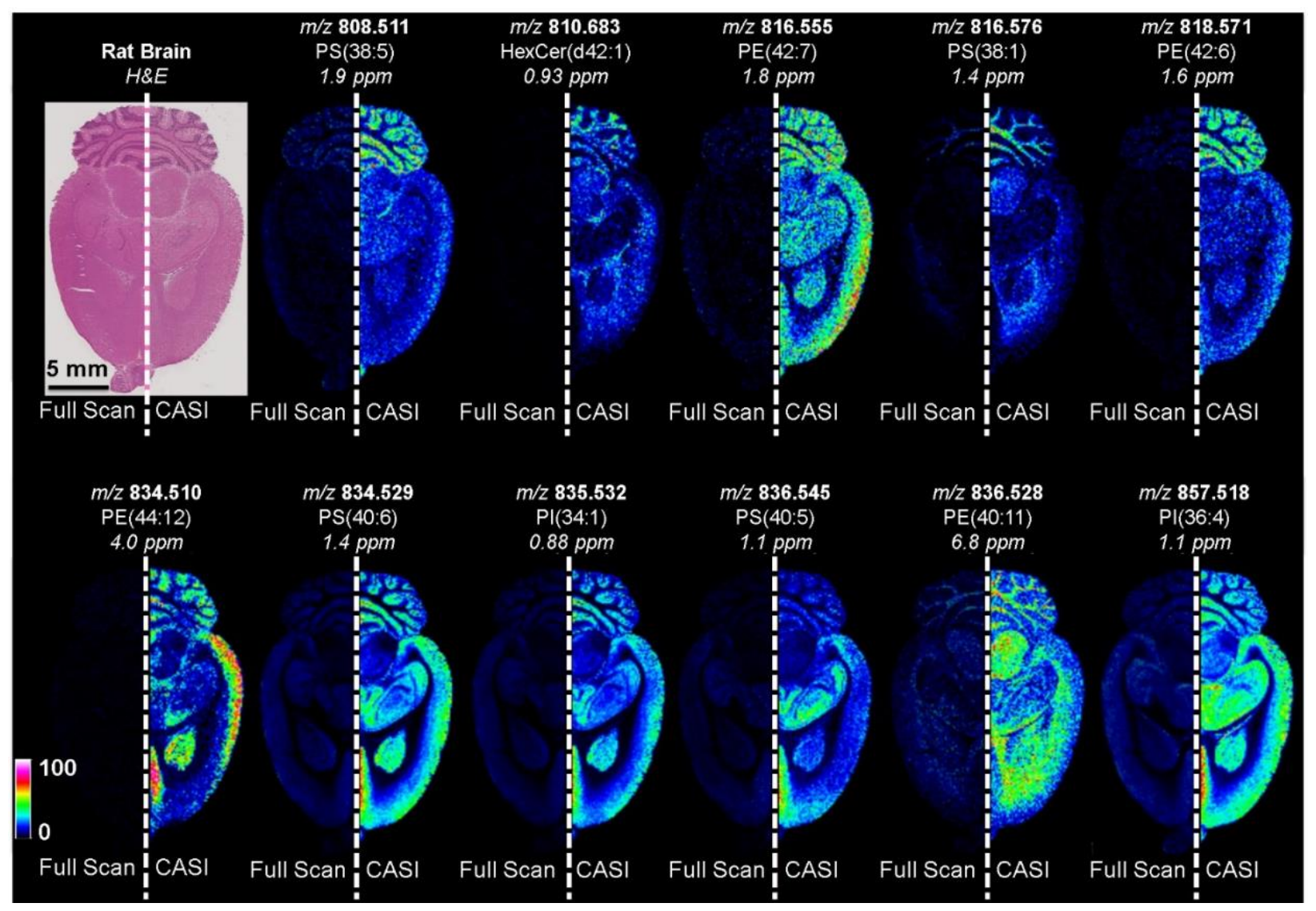

Figure 4: Imaging mass spectrometry analysis of rat brain using (left hemisphere) full scan acquisition mode and (right hemisphere) using a 75 Da CASI window centered at $\mathrm{m} / z$ 845. Ion images for a range of lipids within the CASI window show improved brightness (i.e., sensitivity) and contrast (i.e., dynamic range). Images are shown without normalization.

\section{Selected Ion Ejection}

The ability to selectively eject a small $\mathrm{m} / \mathrm{z}$ interval (Figure 1b) was confirmed by ejecting a single ion ( $m / z$ 588.502) from a red phosphorous distribution (Figure 5). The secular frequency of $m / z 588.502$ in the quadrupole mass filter was calculated based on the dimensionless Mathieu trapping parameters and using the Dehmelt approximation.61-63 Briefly, the known operating conditions of the quadrupole (i.e., physical dimensions, operating frequency, and voltages) allow for the ion secular frequency to be calculated. Using a low-mass cutoff (LMCO) setting of $300 \mathrm{Da}$ in the quadrupole, the secular frequency of $m / z 588.5$ was calculated to be approximately 124.5 
$\mathrm{kHz}$. Almost all of the $m / z 588.502$ ion signal was removed upon using the SIE arbitrary waveform generator to apply a $1.0 \mathrm{~V}, 124.5 \mathrm{kHz}$ AC signal to the quadrupole (Figure 5b). The signal intensities of the neighboring ions in the distribution, $\mathrm{m} / \mathrm{z} 526.554$ and $\mathrm{m} / \mathrm{z} 650.449$, were unaffected, indicating good waveform resolution. The residual $\mathrm{m} / \mathrm{z} 588.502$ ion signal is likely due to the fact that the ion transit time through the quadrupole is relatively short, providing only a limited number of secular oscillations for the ion to come in resonance with the applied signal and be ejected before it reaches the exit of the QMF. Slowing the ion transit through the quadrupole mass filter using a higher gas pressure and/or a lower accelerating lens voltage may enable improved ejection efficiency. Nonetheless, the current result of $\sim 95 \%$ ejection efficiency will be adequate for most applications.

The relative $\mathrm{m} / \mathrm{z}$ width of the ejection window is partially dependent on the frequency of the applied waveforms. The frequency density at larger Mathieu $q$ values is higher than at lower $q$ values, so an AC signal that spans $1 \mathrm{kHz}$ at low frequency (i.e., higher $\mathrm{m} / \mathrm{z}$ ) will encompass a wider range of $\mathrm{m} / \mathrm{z}$ values compared to a $1 \mathrm{kHz}$ wide signal at a higher frequency (i.e., lower $\mathrm{m} / \mathrm{z}$ ). The low-mass cutoff setting on the instrument can be varied by the user to alter the secular frequency of an ion of interest. The relative width of the ejection window is also dependent upon the amplitude of the applied signal. A higher voltage signal may more efficiently eject ions from the quadrupole, but this will also result in a wider $\mathrm{m} / \mathrm{z}$ window. These two parameters, frequency ejection point and ejection amplitude, provide the user with some flexibility in tuning the width of the ejection window. In some applications a wider window may be useful to eject a larger $\mathrm{m} / \mathrm{z}$ range, while in other applications a higher resolution window may be desired to more selectively ejected a smaller mass range. For our purposes, we found that a $1.0 \mathrm{~V}$ signal generally resulted in a 10 Da ejection window with nearly $100 \%$ efficiency. 

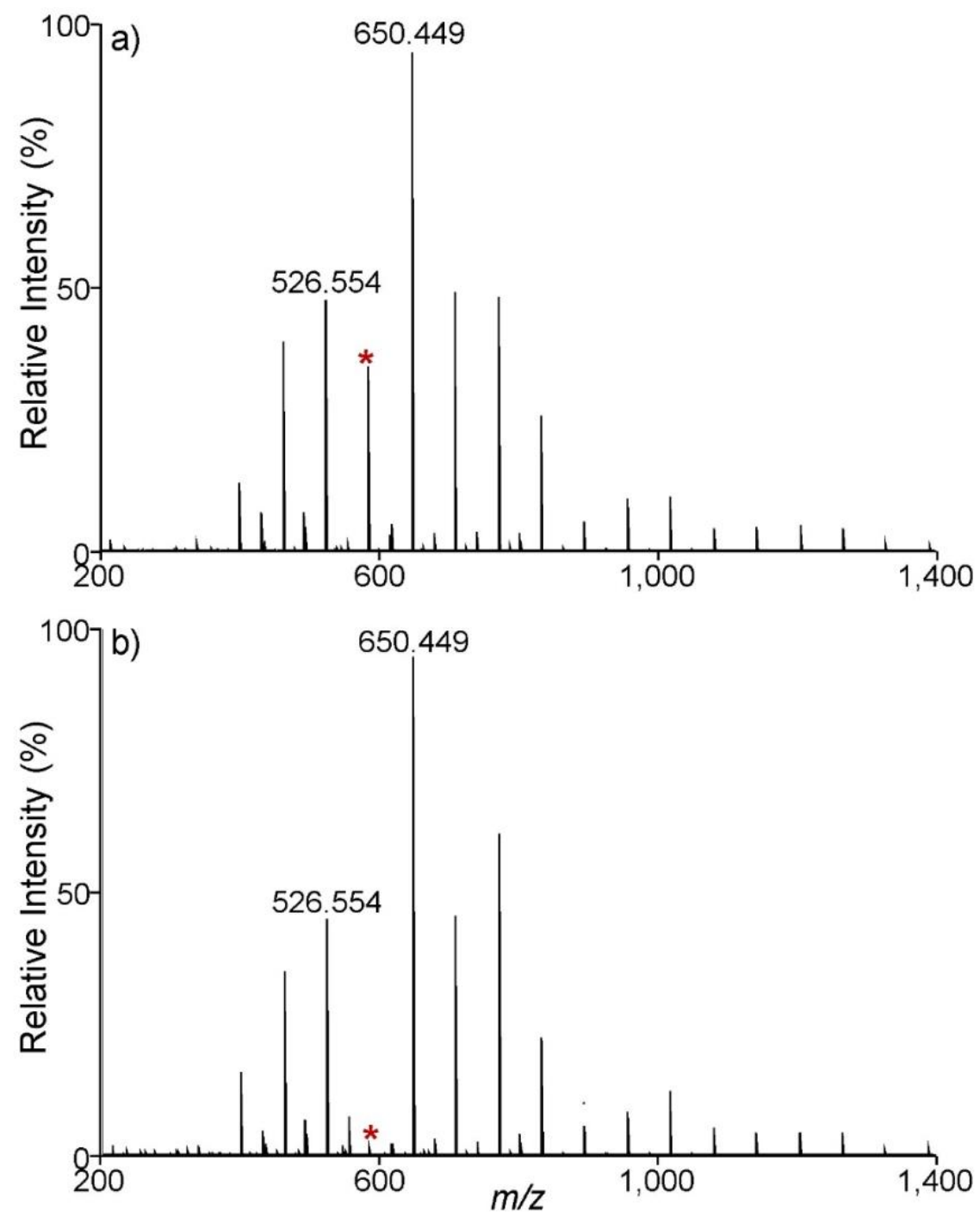

Figure 5: Red phosphorus analysis under (a) off-resonance and (b) selected ion ejection conditions. Selected ion ejection of $\mathrm{m} / \mathrm{z} 588.502$ (red asterisk) in panel (b) was performed using an instrument LMCO of $300 \mathrm{Da}$ and a $1.0 \mathrm{~V}, 124.5 \mathrm{kHz}$ applied waveform, resulting in nearly $100 \%$ ejection efficiency without affecting red phosphorous ions of higher or lower $\mathrm{m} / \mathrm{z}$ values. The ion secular frequency, and thus the frequency of the applied waveform, is calculated using the Mathieu trapping parameters and the Dehmelt approximation. Applying the same voltage waveform offresonance at $129 \mathrm{kHz}$ in panel (a) does affect the intensity of $m / z 588.502$. Spectra shown are averages of 10 scans.

The utility of selected ion ejection for improving sensitivity and dynamic range in an imaging mass spectrometry experiment was evaluated during negative ion mode lipid analysis of 
a rat brain. The lipid $\operatorname{PI}(38: 4)(\mathrm{m} / \mathrm{z} 885.550)$ is frequently observed as the most abundant ion in negative ion mode lipid MALDI IMS experiments.64 There are also several other highly abundant lipid compounds observed in the mass range between $\mathrm{m} / \mathrm{z} 875$ and $\mathrm{m} / \mathrm{z}, 910$, such as the sulfatides SHexCer(40:1) and SHexCer(42:2) (Figure 6a). These ions comprise a significant portion of the charge capacity of the hexapole accumulation cell in this sample, limiting the ability to store other less intense ions. An SIE waveform was applied to the QMF to eject these highly abundant ions during ion transmission (Figure 6b). A $2.5 \mathrm{~V}, 123.5 \mathrm{kHz}$ waveform using a QMF LMCO of 450 Da enabled the ejection of most ions between $\mathrm{m} / \mathrm{z} 875$ and $\mathrm{m} / \mathrm{z}$ 910. A higher voltage SIE waveform was used here compared to that employed in Figure 5 in order to eject ions from a wider $m / z$ range. Comparing the ion images of the lipid ions within the SIE window with and without the waveform applied clearly demonstrates the efficiency of ion ejection (Figure 6c).

The number of laser shots in the SIE and full scan experiments was held constant in Figure 6 to enable a direct comparison of SIE efficiency. However, the removal of the highly abundant ions via SIE indicates that there is now additional unused charge capacity in the hexapole ion trap. This allows for the accumulation of additional laser shots to maximize the intensity of other ions outside of the SIE window without reaching the space charge limit. In Figure 7, a full scan IMS analysis of the rat brain tissue was performed using 55 laser shots (right hemisphere). The accumulation of additional laser shots would breach the space charge limit of the ICR cell and/or the hexapole ion trap. Upon application of the same SIE waveform as used in Figure 6 (2.5 V, 123.5 kHz SIE waveform with a QMF LMCO of $450 \mathrm{Da}$ ), the number of laser shots could be increased to 225 without surpassing the charge capacity of the trap (left hemisphere in Figure 7). Several ions close to the detection limit in the full scan measurement are now observed in SIE experiment. As with the CASI experiment, the improvements in ion intensities with the SIE 
method translate into improvements in image intensity and quality. Many lipids are readily detected with good brightness (i.e., sensitivity) and good image contrast (i.e., dynamic range) in the SIE imaging experiment.
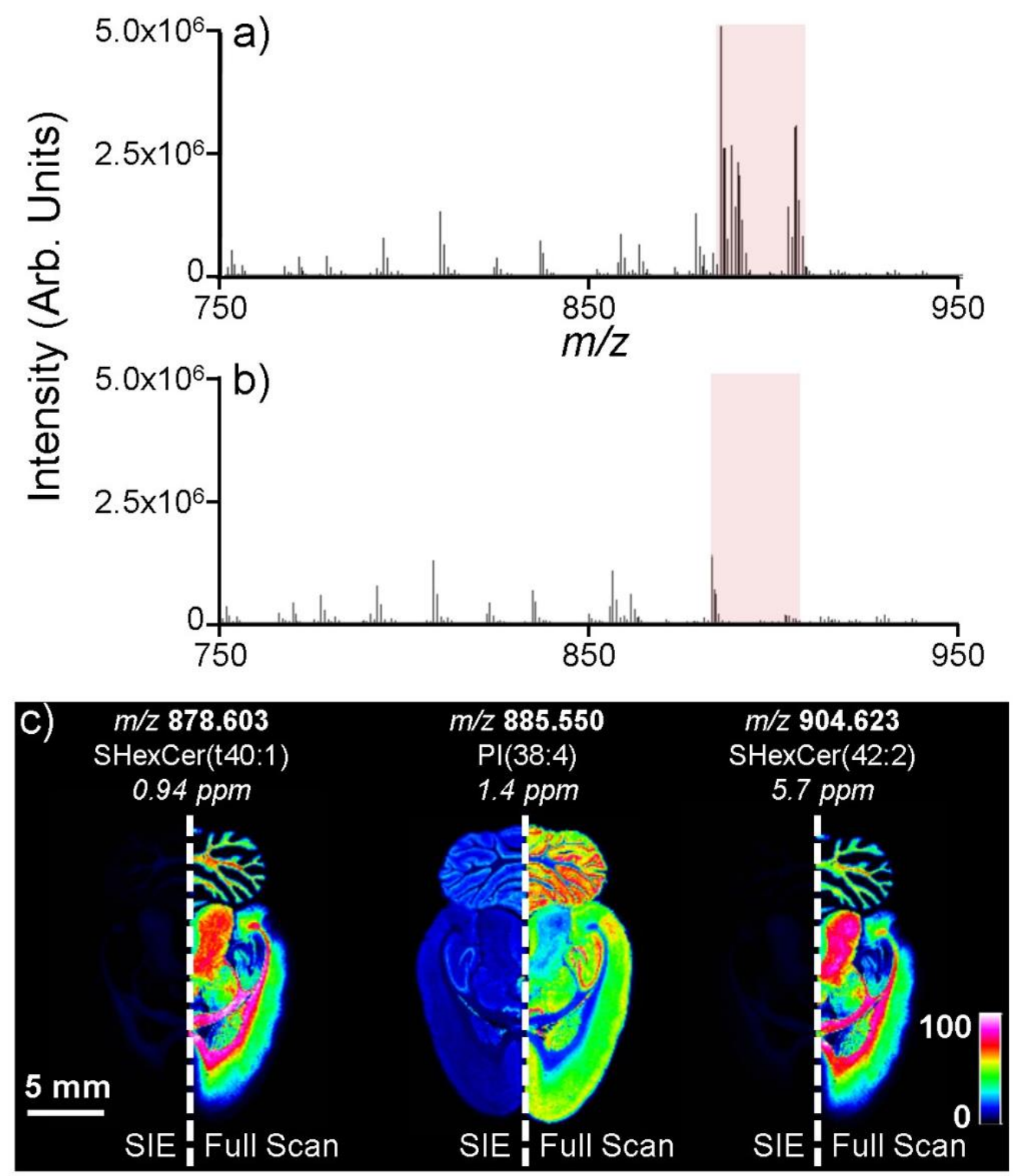

Figure 6: Imaging mass spectrometry analysis of rat brain using (a) full scan acquisition mode and (b) using an SIE method. The SIE method used a $2.5 \mathrm{~V}, 123.5 \mathrm{kHz}$ waveform with a LMCO of $450 \mathrm{Da}$. The number of laser shots (400) was kept constant between the SIE and full scan acquisitions to demonstrate the ability of the SIE waveform to remove most of the ions within the isolation window from the spectrum (highlighted by the red box). c) The applied waveform successfully ejects most lipid ions between $\mathrm{m} / \mathrm{z} 875$ and m/z 910 . 


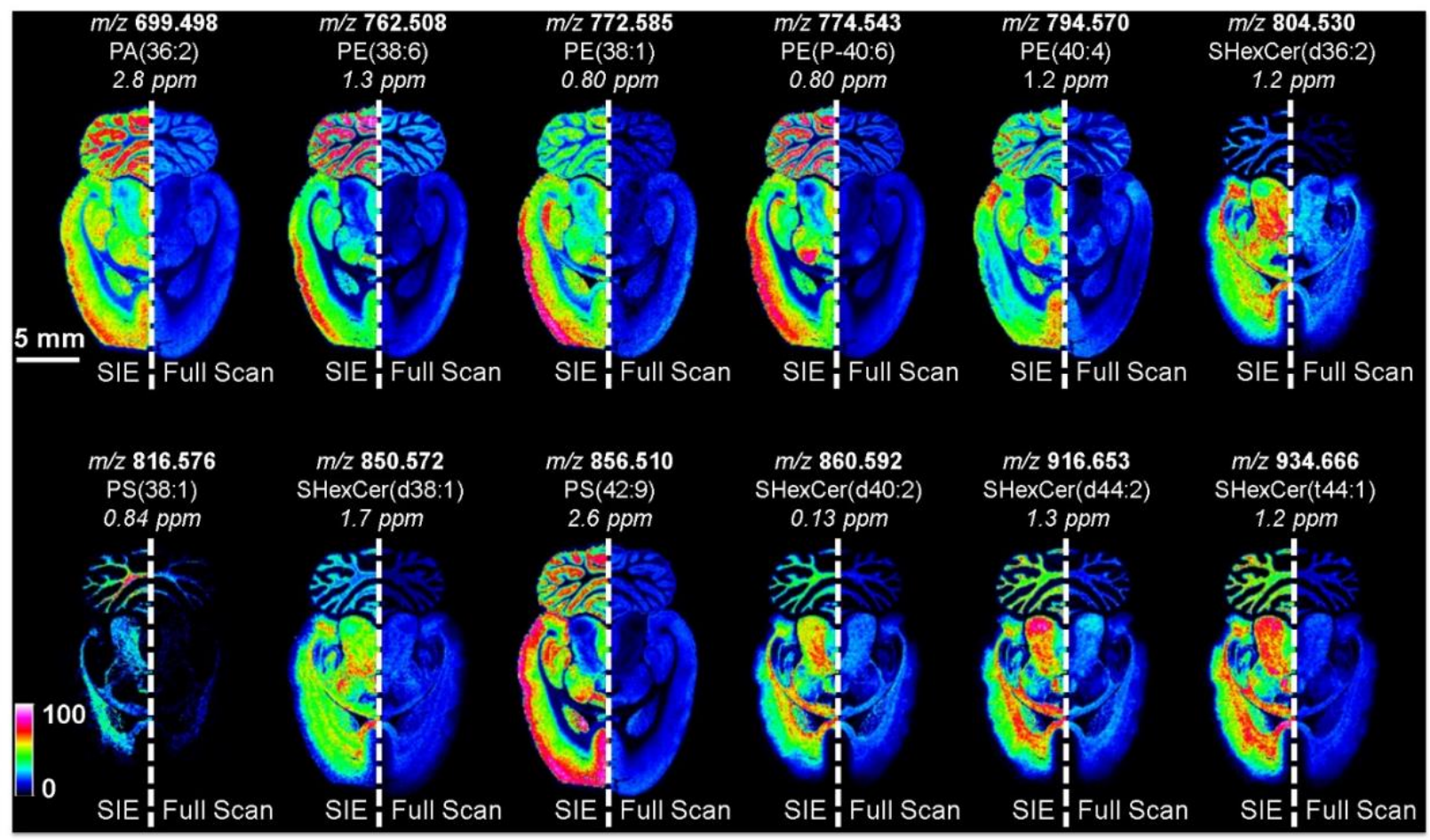

Figure 7: Imaging mass spectrometry analysis of rat brain using (right hemisphere) full scan acquisition mode and (left hemisphere) using a $2.5 \mathrm{~V}, 123.5 \mathrm{kHz}$ SIE waveform with a LMCO of $450 \mathrm{Da}$. The number of laser shots was varied between the two methods to maximize the intensity of the ion populations without reaching the space charge limit (full scan: 55 laser shots, SIE: 225 laser shots). Both experiments were performed using an $80 \mu \mathrm{m}$ random walk. Ion images for a range of lipids show improved brightness (i.e., sensitivity) and contrast (i.e., dynamic range) using the SIE acquisition method. Images are shown without normalization.

\section{CONCLUSIONS}

We have characterized two methods of ion enrichment for use during imaging mass spectrometry experiments. Continuous accumulation of selected ions (CASI) and selected ion ejection (SIE) are both rapid gas-phase strategies that fractionate ions based on their $\mathrm{m} / \mathrm{z}$ ratios. CASI is performed by using the RF/DC isolation feature of the quadrupole mass filter to restrict ion transmission to a small $\mathrm{m} / \mathrm{z}$ range, allowing for the targeted enrichment of ions trapped downstream in the hexapole ion trap by over 25 -fold. SIE is performed by using a supplementary AC waveform applied to the quadrupole mass filter to selectively eject ions within a small $\mathrm{m} / z$ range, allowing for the broadband enrichment of ions trapped downstream in the hexapole ion trap 
by over 4-fold. Both of these methods operate by discarding signal from unwanted ions in order to maximize the ion population for compounds of interest. Both CASI and SIE are enabled with no impact on instrument throughput, allowing for the acquisition of ion images with improved brightness (i.e., sensitivity) and contrast (i.e., dynamic range) without requiring longer analysis times. CASI methods are useful in targeted applications where analytes of interest fall within a small mass range (i.e., a drug and its metabolite), while SIE methods are more beneficial in untargeted or exploratory workflows where it is desirable to increase the sensitivity of lower abundance molecules across a broad mass range.

\section{ACKNOWLEDGEMENTS}

This work was supported by the National Institutes of Health (NIH) under award P41 GM103391 (National Institute of General Medical Sciences [NIGMS]). The authors would also like to thank Dr. Ryan Danell of Danell Consulting, Inc. as well as Dr. Christian Berg of Bruker Daltonics for input regarding the quadrupole modifications. 


\section{REFERENCES}

1. Bennett, B. D.; Kimball, E. H.; Gao, M.; Osterhout, R.; Van Dien, S. J.; Rabinowitz, J. D., Absolute metabolite concentrations and implied enzyme active site occupancy in Escherichia coli. Nat. Chem. Biol. 2009, 5 (8), 593-599.

2. Schmitt-Kopplin, P.; Frommberger, M., Capillary electrophoresis - mass spectrometry: 15 years of developments and applications. Electrophoresis 2003, 24 (22-23), 3837-3867.

3. Dettmer, K.; Aronov, P. A.; Hammock, B. D., Mass spectrometry-based metabolomics. 2007, 26 (1), 51-78.

4. Yates, J. R.; Ruse, C. I.; Nakorchevsky, A., Proteomics by mass spectrometry: approaches, advances, and applications. 2009, 11 (1), 49-79.

5. Blanksby, S. J.; Mitchell, T. W., Advances in mass spectrometry for lipidomics. 2010, 3 (1), 433-465.

6. Holmes, J. C.; Morrell, F. A., Oscillographic mass spectrometric monitoring of gas chromatography. Appl. Spectrosc. 1957, 11 (2), 86-87.

7. Gohlke, R. S., Time-of-flight mass spectrometry and gas-liquid partition chromatography. Anal. Chem. 1959, 31 (4), 535-541.

8. Tal'roze, V. L.; Karpov, G. V.; Gorodetski, I. G.; Skurat, V. E., Capillary system for the introduction of liquid mixtures into an analytical mass spectrometer. Russian Journal of Physical Chemistry 1968, 42, 1658-1664.

9. Baldwin, M. A.; McLafferty, F. W., Liquid chromatography-mass spectrometry interface-I: The direct introduction of liquid solutions into a chemical ionization mass spectrometer. Org. Mass Spectrom. 1973, 7 (9), 1111-1112.

10. Olivares, J. A.; Nguyen, N. T.; Yonker, C. R.; Smith, R. D., On-line mass spectrometric detection for capillary zone electrophoresis. Anal. Chem. 1987, 59 (8), 1230-1232.

11. Pu, F.; Chiang, S.; Zhang, W.; Ouyang, Z., Direct sampling mass spectrometry for clinical analysis. Analyst 2019, 144 (4), 1034-1051.

12. Caprioli, R. M.; Farmer, T. B.; Gile, J., Molecular imaging of biological samples: localization of peptides and proteins using MALDI-TOF MS. Anal. Chem. 1997, 69, 4751-4760.

13. McDonnell, L. A.; Heeren, R. M. A., Imaging mass spectrometry. Mass Spectrom. Rev. 2007, 26 (4), 606-643.

14. Norris, J. L.; Caprioli, R. M., Analysis of tissue specimens by matrix-assisted laser desorption/ionization imaging mass spectrometry in biological and clinical research. Chem. Rev. 2013, 113 (4), 2309-2342.

15. Wu, C. P.; Dill, A. L.; Eberlin, L. S.; Cooks, R. G.; Ifa, D. R., Mass spectrometry imaging under ambient conditions. Mass Spectrom. Rev. 2013, 32 (3), 218-243.

16. Cornett, D. S.; Frappier, S. L.; Caprioli, R. M., MALDI-FTICR imaging mass spectrometry of drugs and metabolites in tissue. Anal. Chem. 2008, 80 (14), 5648-5653.

17. Lemaire, R.; Desmons, A.; Tabet, J.; Day, R.; Salzet, M.; Fournier, I., Direct analysis and MALDI imaging of formalin-fixed, paraffin-embedded tissue sections. J. Proteome Res. 2007, 6 (4), 1295-1305.

18. Manier, M.; Reyzer, M.; Goh, A.; Dartois, V.; Via, L.; Barry, C.; Caprioli, R., Reagent precoated targets for rapid in-tissue derivatization of the anti-tuberculosis drug isoniazid followed by MALDI imaging mass spectrometry. J. Am. Soc. Mass Spectrom. 2011, 22 (8), 1409-1419. 
19. Yang, J.; Caprioli, R. M., Matrix sublimation/recrystallization for imaging proteins by mass spectrometry at high spatial resolution. Anal. Chem. 2011, 83 (14), 5728-5734.

20. Angel, P. M.; Spraggins, J. M.; Baldwin, H. S.; Caprioli, R. M., Enhanced sensitivity for high spatial resolution lipid analysis by negative ion mode matrix assisted laser desorption ionization imaging mass spectrometry. Anal. Chem. 2012, 84 (3), 1557-1564.

21. Shariatgorji, M.; Nilsson, A.; Goodwin, R.; Källback, P.; Schintu, N.; Zhang, X.; Crossman, A.; Bezard, E.; Svenningsson, P.; Andren, P., Direct targeted quantitative molecular imaging of neurotransmitters in brain tissue sections. Neuron 2014, 84 (4), 697-707.

22. Toue, S.; Sugiura, Y.; Kubo, A.; Ohmura, M.; Karakawa, S.; Mizukoshi, T.; Yoneda, J.; Miyano, H.; Noguchi, Y.; Kobayashi, T.; Kabe, Y.; Suematsu, M., Microscopic imaging mass spectrometry assisted by on-tissue chemical derivatization for visualizing multiple amino acids in human colon cancer xenografts. Proteomics 2014, 14 (7-8), 810-819.

23. Shariatgorji, M.; Nilsson, A.; Kallback, P.; Karlsson, O.; Zhang, X. Q.; Svenningsson, P.; Andren, P. E., Pyrylium Salts as Reactive Matrices for MALDI-MS Imaging of Biologically Active Primary Amines. J. Am. Soc. Mass Spectrom. 2015, 26 (6), 934-939.

24. Kanu, A. B.; Dwivedi, P.; Tam, M.; Matz, L.; Hill Jr, H. H., Ion mobility-mass spectrometry. J. Mass Spectrom. 2008, 43 (1), 1-22.

25. Ruotolo, B. T.; Gillig, K. J.; Stone, E. G.; Russell, D. H., Peak capacity of ion mobility mass spectrometry:: Separation of peptides in helium buffer gas. Journal of Chromatography $B$ 2002, 782 (1), 385-392.

26. Lapthorn, C.; Pullen, F.; Chowdhry, B. Z., Ion mobility spectrometry-mass spectrometry (IMS-MS) of small molecules: Separating and assigning structures to ions. Mass Spectrom. Rev. 2013, 32 (1), 43-71.

27. Wang, Y.; Zhang, X.; Zhai, Y.; Jiang, Y.; Fang, X.; Zhou, M.; Deng, Y.; Xu, W., Mass selective ion transfer and accumulation in ion trap arrays. Anal. Chem. 2014, 86 (20), 10164-10170.

28. Quenzer, T. L.; Emmett, M. R.; Hendrickson, C. L.; Kelly, P. H.; Marshall, A. G., High sensitivity Fourier transform ion cyclotron resonance mass spectrometry for biological analysis with nano-LC and microelectrospray ionization. Anal. Chem. 2001, 73 (8), 1721-1725.

29. Senko, M. W.; Hendrickson, C. L.; Emmett, M. R.; Shi, S. D. H.; Marshall, A. G., External accumulation of ions for enhanced electrospray ionization fourier transform ion cyclotron resonance mass spectrometry. J. Am. Soc. Mass Spectrom. 1997, 8 (9), 970-976. 30. Hager, J. W., A new linear ion trap mass spectrometer. Rapid Commun. Mass Spectrom. 2002, 16, 512-526.

31. Pekar Second, T.; Blethrow, J. D.; Schwartz, J. C.; Merrihew, G. E.; MacCoss, M. J.; Swaney, D. L.; Russell, J. D.; Coon, J. J.; Zabrouskov, V., Dual-pressure linear ion trap mass spectrometer improving the analysis of complex protein mixtures. Anal. Chem. 2009, 81 (18), 7757-7765.

32. Wang, Y.; Shi, S. D. H.; Hendrickson, C. L.; Marshall, A. G., Mass-selective ion accumulation and fragmentation in a linear octopole ion trap external to a fourier transform ion cyclotron resonance mass spectrometer. Int. J. Mass Spectrom. 2000, 198 (1), 113-120.

33. Belov, M. E.; Nikolaev, E. N.; Anderson, G. A.; Auberry, K. J.; Harkewicz, R.; Smith, R. D., Electrospray ionization-fourier transform ion cyclotron mass spectrometry using ion preselection and external accumulation for ultrahigh sensitivity. J. Am. Soc. Mass Spectrom. 2001, 12 (1), 38-48. 
34. Belov, M. E.; Anderson, G. A.; Angell, N. H.; Shen, Y.; Tolic, N.; Udseth, H. R.; Smith, R. D., Dynamic range expansion applied to mass spectrometry based on data-dependent selective ion ejection in capillary liquid chromatography Fourier transform ion cyclotron resonance for enhanced proteome characterization. Anal. Chem. 2001, 73 (21), 5052-5060. 35. Campbell, J. M.; Collings, B. A.; Douglas, D. J., A new linear ion trap time-of-flight system with tandem mass spectrometry capabilities. 1998, 12 (20), 1463-1474.

36. Cha, B.; Blades, M.; Douglas, D. J., An interface with a linear quadrupole ion guide for an electrospray-ion trap mass spectrometer system. Anal. Chem. 2000, 72 (22), 5647-5654. 37. Collings, B. A.; Douglas, D. J., Observation of higher order quadrupole excitation frequencies in a linear ion trap. J. Am. Soc. Mass Spectrom. 2000, 11 (11), 1016-1022.

38. Jackson, S. N.; Ugarov, M.; Egan, T.; Post, J. D.; Langlais, D.; Schultz, J. A.; Woods, A. S., MALDI-ion mobility-TOFMS imaging of lipids in rat brain tissue. J. Mass Spectrom. 2007, 42 (8), 1093-1098.

39. McLean, J. A.; Ridenour, W. B.; Caprioli, R. M., Profiling and imaging of tissues by imaging ion mobility-mass spectrometry. J. Mass Spectrom. 2007, 42 (8), 1099-1105.

40. Trim, P. J.; Henson, C. M.; Avery, J. L.; McEwen, A.; Snel, M. F.; Claude, E.; Marshall, P. S.; West, A.; Princivalle, A. P.; Clench, M. R., Matrix-assisted laser desorption/ionization-ion mobility separation-mass spectrometry imaging of vinblastine in whole body tissue sections. Anal. Chem. 2008, 80 (22), 8628-8634.

41. Stauber, J.; MacAleese, L.; Franck, J.; Claude, E.; Snel, M.; Kaletas, B.; Wiel, I. M. V. D.; Wisztorski, M.; Fournier, I.; Heeren, R. M. A., On-tissue protein identification and imaging by MALDI-ion mobility mass spectrometry. J. Am. Soc. Mass Spectrom. 2010, 21 (3), 338-347.

42. Jackson, S. N.; Barbacci, D.; Egan, T.; Lewis, E. K.; Schultz, J. A.; Woods, A. S., MALDI-ion mobility mass spectrometry of lipids in negative ion mode. Analytical Methods 2014, 6 (14), 5001-5007.

43. Sans, M.; Feider, C. L.; Eberlin, L. S., Advances in mass spectrometry imaging coupled to ion mobility spectrometry for enhanced imaging of biological tissues. Curr. Opin. Chem. Biol. 2018, 42, 138-146.

44. Anderson, D. M. G.; Mills, D.; Spraggins, J.; Lambert, W. S.; Calkins, D. J.; Schey, K. L., High-resolution matrix-assisted laser desorption ionization-imaging mass spectrometry of lipids in rodent optic nerve tissue. Mol Vis 2013, 19, 581-592.

45. Groseclose, M. R.; Castellino, S., A Mimetic Tissue Model for the Quantification of Drug Distributions by MALDI Imaging Mass Spectrometry. Anal. Chem. 2013, 85 (21), 1009910106.

46. Sun, N.; Walch, A., Qualitative and quantitative mass spectrometry imaging of drugs and metabolites in tissue at therapeutic levels. Histochemistry and Cell Biology 2013, 140 (2), 93104.

47. Cobice, D. F.; Goodwin, R. J. A.; Andren, P. E.; Nilsson, A.; Mackay, C. L.; Andrew, R., Future technology insight: mass spectrometry imaging as a tool in drug research and development. Br. J. Pharmacol. 2015, 172 (13), 3266-3283.

48. Wenke, J. L.; Rose, K. L.; Spraggins, J. M.; Schey, K. L., MALDI imaging mass spectrometry spatially maps age-related deamidation and truncation of human lens aquaporin- 0 . Investigative Ophthalmology \& Visual Science 2015, 56 (12), 7398-7405.

49. Hulme, H. E.; Meikle, L. M.; Wessel, H.; Strittmatter, N.; Swales, J.; Thomson, C.; Nilsson, A.; Nibbs, R. J. B.; Milling, S.; Andren, P. E.; Mackay, C. L.; Dexter, A.; Bunch, J.; 
Goodwin, R. J. A.; Burchmore, R.; Wall, D. M., Mass spectrometry imaging identifies palmitoylcarnitine as an immunological mediator during Salmonella Typhimurium infection. Scientific Reports 2017, 7 (1), 2786.

50. Yang, B.; Patterson, N. H.; Tsui, T.; Caprioli, R. M.; Norris, J. L., Single-cell mass spectrometry reveals changes in lipid and metabolite expression in RAW 264.7 cells upon lipopolysaccharide stimulation. J. Am. Soc. Mass Spectrom. 2018, 29 (5), 1012-1020.

51. Prentice, B. M.; Hart, N. J.; Phillips, N.; Haliyur, R.; Judd, A.; Armandala, R.; Spraggins, J. M.; Lowe, C. L.; Boyd, K. L.; Stein, R. W.; Wright, C. V.; Norris, J. L.; Powers, A. C.; Brissova, M.; Caprioli, R. M., Imaging mass spectrometry enables molecular profiling of mouse and human pancreatic tissue. Diabetologia 2019, 62, 1036-1047.

52. Kostyukevich, Y. I.; Vladimirov, G. N.; Nikolaev, E. N., Dynamically harmonized FTICR cell with specially shaped electrodes for compensation of inhomogeneity of the magnetic field. Computer simulations of the electric field and ion motion dynamics. J. Am. Soc. Mass Spectrom. 2012, 23 (12), 2198-2207.

53. Folch, J.; Lees, M.; Stanley, H. S., A simple method for the isolation and purification of total lipids from animal tissues. Journal of Biological Chemistry 1957, 226, 497-509.

54. Hankin, J. A.; Barkley, R. M.; Murphy, R. C., Sublimation as a method of matrix application for mass spectrometric imaging. J. Am. Soc. Mass Spectrom. 2007, 18 (9), 16461652.

55. Thomas, A.; Charbonneau, J.; Fournaise, E.; Chaurand, P., Sublimation of new matrix candidates for high spatial resolution imaging mass spectrometry of lipids: enhanced information in both positive and negative polarities after 1,5-diaminonapthalene deposition. Anal. Chem. 2012, 84 (4), 2048-2054.

56. Strohalm, M.; Hassman, M.; Kosata, B.; Kodicek, M., mMass data miner: an open source alternative for mass spectrometric data analysis. Rapid Commun. Mass Spectrom. 2008, 22 (6), 905-908.

57. Paša-Tolić, L.; Huang, Y.; Guan, S.; Kim, H. S.; Marshall, A. G., Ultrahigh-resolution matrix-assisted laser desorption/ionization Fourier transform ion cyclotron resonance mass spectra of peptides. 1995, 30 (6), 825-833.

58. Mitchell, D. W.; Smith, R. D., Prediction of a space charge induced upper molecular mass limit towards achieving unit mass resolution in Fourier transform ion cyclotron resonance mass spectrometry. 1996, 31 (7), 771-790.

59. Marshall, A. G.; Hendrickson, C. L.; Jackson, G. S., Fourier transform ion cyclotron resonance mass spectrometry: A primer. 1998, 17 (1), 1-35.

60. Nikolaev, E. N.; Kostyukevich, Y. I.; Vladimirov, G. N., Fourier transform ion cyclotron resonance (FT ICR) mass spectrometry: Theory and simulations. 2016, 35 (2), 219-258.

61. Mathieu, E., Mémoire sur le mouvement vibratoire d'une membrane de forme elliptique. J. Math. Pures Appl. 1868, 13, 137-203.

62. McLachlan, N. W., Theory and Application of Mathieu Functions. Clarendon Press: Oxford, 1947.

63. March, R. E.; Todd, J. F. J., Quadrupole ion trap mass spectrometry. 2nd ed.; J. Wiley: Hoboken, N.J., 2005; p xxxv, 346 p.

64. Zemski Berry, K. A. H., Joseph A.; Barkley, Robert A.; Spraggins, Jeffery M.; Caprioli, Richard M.; Murphy, Robert C., MALDI imaging of lipid biochemistry in tissues by mass spectrometry. Chem. Rev. 2011, 111 (10), 6491-6512. 